ARTÍCULOS ORIGINALES

Rev Chil Salud Pública 2019,

Vol 23(1): 31-41

\title{
SUICIDIO Y DEPRESIÓN EN ADOLESCENTES: UNA REVISIÓN DE LA LITERATURA
}

\author{
SUICIDE AND DEPRESSION IN ADOLESCENTS: \\ A LITERATURE REVIEW
}

\section{RESUMEN}

Antecedentes: Aunque el suicidio es un problema secular de la salud pública, la reciente preocupación es la edad y magnitud de ocurrencia, particularmente al asociarse a depresión. Objetivo: Revisar el estado del arte sobre el fenómeno del suicidio, sus vínculos con depresión en población adolescente y de adultos jóvenes e identificar los principales aspectos asociados, con el propósito de plantear estrategias de prevención. Método: Se realizó una revisión de la literatura usando las bases de datos científicas Scielo, Redalyc y Pubmed y los descriptores adolescencia, jóvenes, depresión, intento suicida, conducta suicida, suicidio, tanto en casteIlano como en inglés. Se incluyó un total de 53 artículos publicados entre 2013 y 2017 y se atendió fundamentalmente a la epidemiología y los factores asociados. Resultados: Se reporta un amplio rango de prevalencia en el mundo, que va de 2.4 (Egipto) hasta 46.5 (Lituania) suicidas de entre 15 y 29 años por cada 100 mil habitantes, con un predominio de hombres. Además, se registra ideación suicida en hasta un tercio de la población menor de 20 años. Se reportan asociaciones con atributos de personalidad, adicciones, ambientes familiares y sociales hostiles, y carencia de credo religioso. Discusión: El suicidio en jóvenes se percibe vinculado a violencia de tipo estructural, lo cual obliga a reflexionar sobre las opciones viables de desarrollo humano.

Palabras Clave: Suicidio, jóvenes, Trastornos anímicos, Factores de riesgo

Gerardo de Jesús Moreno-Gordillo Facultad de Medicina Humana Dr. Manuel Velasco Suárez, UNACH

Laura E. Trujillo-Olivera Doctora en Ciencias. El Colegio de la Frontera Sur, Unidad SCLC elena2_333@hotmail.com

Néstor R. García-Chong Doctor en Ciencias. Hospital de Especialidades Pediátricas (HEP) del Centro Regional de Alta Especialidad de Chiapas (CRAE), Facultad de Medicina Humana, Dr. Manuel Velasco Suárez, UNACH

Fernando Tapia-Garduño Terapeuta Familiar. Hospital de Especialidades Pediátricas (HEP) del Centro Regional de Alta Especialidad de Chiapas (CRAE)

Artículo recibido el 13/04/2018. Aceptado el 31/07/2018

\section{ABSTRACT}

Background: Although suicide has long been a public health concern, recent attention has focused on its elevated prevalence, particularly when associated with depression, and the age of the affected individuals. Objective: To review literature related to the phenomenon of suicide and its association with depression in the adolescent population and to identify the principal associated factors, in order to propose prevention strategies. Methods: A literature review was conducted using the databases Scielo, Redalyc and Pubmed and the keywords adolescence, youth, depression, suicide attempt, suicidal behavior, and suicide, in Spanish and in English. A total of 53 articles, published between 2013 and 2017, were included, and the analysis focused on the phenomenon's epidemiology and related associated factors. Results: Suicide prevalence rates range widely worldwide, from 2.4 (Egypt) to 46.5 (Lithuania) per 100,00 inhabitants between 15 and 29 years of age, affecting more men than women. Additionally, approximately a third of individuals under 20 years of age have presented suicide ideation. There are reported associations between suicide and personality attributes, addictions, hostile family and social environments, and the absence of religious beliefs. Discussions: Suicide in young adults is perceived to be linked to structural violence, which necessitates the exploration of viable options for human development.

Keywords: Suicide, Youth, Mood disorders, Risk factors. 


\section{INTRODUCCIÓN}

La adolescencia es una época determinante para el desarrollo de los seres humanos, cuyas experiencias modulan la formación del adulto, generándose cambios en diversos ámbitos para lograr independencia, identidad y aceptación social. Es el periodo más sano desde el punto de vista orgánico, pero es una etapa de alto riesgo, ya que la mayoría de los problemas de salud son consecuencia de comportamientos iniciados en esta época: consumo de drogas, actividades sexuales de riesgo, actos de violencia y accidentes ${ }^{1}$.

Uno de cada cinco adolescentes en el mundo tiene algún problema de salud mental2,3. Depresión es la principal causa de discapacidad en la mayoría de los países; el 85\% residen en países en vías de desarrollo ${ }^{4,5}$.

De acuerdo con el Manual Diagnóstico y Estadístico de Trastornos Mentales (DSM 5) la depresión es un trastorno mental que consiste en diversos síntomas como pérdida de la energía y del interés, cambios del apetito, problemas para concentrarse, sentimientos de tristeza, trastornos del sueño, anhedonia, auto culpabilidad, cambios de peso y agitación, que pueden relacionarse con otros trastornos ${ }^{6}$. La versión beta de la CIE-11 ofrece una única categoría diagnóstica, «trastornos del ánimo», subdividida en trastornos bipolares y trastornos depresivos. El DSM 5 crea dos secciones diferenciadas, una para los «trastornos depresivos» y otra para los «trastornos bipolares y relacionados ${ }^{7}$.

En Latinoamérica la depresión es el segundo trastorno mental más común después del alcoholismo8. La depresión, mala cohesión familiar y farmacodependencia son determinantes de salud mental para el suicidio; asimismo el neuroticismo es un factor de riesgo para el suicidio en problemas familiares y amorosos $^{9,10}$.

Según OMS, en 2012 ocurrieron aproximadamente 804,000 suicidios en todo el mundo, representando una tasa anual de 11.4 suicidios por cada 100,000 habitantes, 15 en hombres y 8 en mujeres de la población en general ${ }^{10-13}$.

La incidencia de suicidios consumados se ha incrementado recientemente. En México durante 1970 fue de 1.1 casos/100 000 habitantes y en el 2007 se reportaron 4.2 defunciones por la misma constante poblacional, con mayor afectación en me- nores de 20 años. Entre 1998 y 2011 se registraron 146,295 defunciones por lesión de causa externa en menores de 20 años; el 7\% correspondió a suicidios empleando con más frecuencia el ahorcamiento y armas de fuego en hombres y ahorcamiento y envenenamiento en mujeres ${ }^{14,11}$. Durante el periodo la tasa de suicidios se elevó (hombres de 26.6 a 42.5; mujeres de 10.4 a 21.1) en adolescentes por millón de habitantes en general ${ }^{14}$.

Ideación suicida se refiere a pensamientos intrusivos y repetitivos sobre la muerte autoinflingida, sobre formas, objetos y circunstancias deseadas de morir ${ }^{15}$. Las estadísticas pueden parecer bajas, pero en población joven ha ido en aumento. Durante la década comprendida entre 1990 y 2000 en México aumentó el $150 \%$, reportándose que $40 \%$ de los suicidas han intentado varias veces. Sin embargo, el intento representa un paso en este proceso que empieza con ideación, continúa con el pensamiento, el intento y por último la consumación ${ }^{16}$.También se identifica que la orientación suicida antecede a la ideación ya que es la percepción de que la vida no es útil y que sería mejor estar muerto ${ }^{17}$. $\mathrm{Al}$ asociarse a depresión, se reporta que la mejoría genera la energía suficiente para avanzar al intento suicida.

La interacción humana en la adolescencia, más compleja en la actualidad, genera un sustrato favorable para los estados depresivos. La violencia, en todas sus formas (psicológica [etiquetas]; física [buIlyng]; sexual entre las más comunes, sin ser las únicas) puede causar en la víctima el aumento de síntomas, en pacientes depresivos sólo aparecen rabia e incomodidad cuando son etiquetados ${ }^{18}$; asimismo se reporta un riesgo aumentado de presentar problemas de salud mental incluyendo ideación e intento de suicidio, que puede persistir en la adultez. Cerca del 38\% de estudiantes entre 13-15 años de edad de Latinoamérica han sufrido bullying en el último mes $^{19}$. El consumo de tabaco, alcohol y estupefacientes se mencionan vinculadas a sintomatología depresiva.

La depresión puede ser el resultado de un trauma sufrido por la separación familiar y esto dificulta el establecimiento de nuevos vínculos, convirtiéndose en un círculo vicioso ${ }^{20}$.

En México se reporta que tener una autoestima baja y el consumo de drogas duplican el riesgo de sintomatología depresiva; las entidades en que 
predomina mayor prevalencia en adolescentes son Guerrero, Puebla, Michoacán y Tabasco que presentaron 34\%, en contraste con Chiapas que solo presentó el $16 \%{ }^{21}$.

La prevalencia en EUA, Europa y Brasil son más elevadas ya que más del $15 \%$ de la población ha padecido depresión. En Colombia y Chile, la situación es muy similar a la de México. En México, menos de $20 \%$ de quienes presentan un trastorno afectivo buscan algún tipo de ayuda. Entre las variables psicosociales asociadas con la depresión destacan: a) ser mujer, dedicarse únicamente a las labores del hogar, cuidar a algún enfermo; b) bajo nivel socioeconómico; c) hombres desempleados; d) aislamiento social; e) problemas legales; f) experiencias de violencia; g) consumir sustancias adictivas; $y$, h) migración ${ }^{22}$.

El suicidio ocupa el quinto puesto en causas de mortalidad, en chicos de 5-14 años y el tercero entre los de 15-24 años ${ }^{23,24}$. Actualmente el uso de psicofármacos no tiene un impacto significativo en la disminución de las conductas suicidas y depresión. Más del 90\% de quienes intentan el suicidio presentan un trastorno psiquiátrico subyacente. La principal forma de prevenirlo es tratando la patología de base ${ }^{24}$. Se reporta que el $27 \%$ de los intentos de suicidio son debidos a la depresión ${ }^{25}$.

Considerando que la adolescencia es una etapa vital trascendente para las sociedades, el propósito de este trabajo es revisar la situación reportada (Iínea base) sobre el suicidio y la depresión e identificar aspectos vinculados en jóvenes para replantear estrategias de intervención en salud mental, particularmente en México.

\section{MÉTODO}

Se trata de un estudio de revisión de la literatura publicada de 5 años a la fecha a excepción de Arrona-Palacios ${ }^{26}$ que es quien define qué es un suicidio consumado. En este trabajo se consideraron las bases de datos de Scielo, Redalyc y Pubmed, de las cuales se seleccionaron 22, 17 y 13 artículos, respectivamente.

Para su localización, se buscó la combinación de los siguientes descriptores: Depresión, Suicidio, Adolescentes, Factores de riesgo, Genética. Para su selección se tomaron en cuenta los siguientes criterios de inclusión: publicaciones entre los años 2013 y 2017, publicaciones en inglés o en español que incluyeran cualquiera de las siguientes palabras o descriptores en su título: adolescentes, adolescencia, depresión, suicidio, genético y psicosocial.

Los criterios de exclusión fueron: investigaciones no publicadas a texto completo, tesis, trabajos en idiomas distintos al inglés o al español, o que involucraran otros trastornos psiquiátricos distintos a depresión y suicidio como factores asociados. Tampoco se consideraron trabajos comprendidos como literatura gris.

Para la elaboración de las tablas se tomaron en cuenta los artículos que establecían edades comprendidas entre 10-19 años así como prevalencia de comportamientos suicidas y los factores relacionados y de riesgo en sus resultados. Fueron incluidos algunos artículos, en que se excedían las edades de los participantes, debido a que presentaban elementos relevantes y prevalencia claramente determinada de los eventos en estudio.

Los autores reconocen que los artículos incluidos reportan diferentes etapas del suicidio, así como diferentes edades de los participantes, de igual manera que éstos se encontraban en condiciones clínicas heterogéneas, por lo que se consideró resumirlos por grupos para su presentación.

\section{RESULTADOS}

\section{Prevalencia}

Estudios sobre suicidio reportan mayor frecuencia en mujeres (2:1) con respecto al intento; lo inverso respecto a suicidios consumados. Las mujeres intentan más veces suicidarse pero fracasan $27,28,15$, mientras los hombres utilizan métodos más violentos y efectivos (armas, ahorcamiento). Las mujeres utilizan ahorcamiento y envenenamiento, asimismo suelen pedir ayuda más comúnmente que los hombres $^{29}$.

En la tabla 1 se puede observar la prevalencia reportada de ideación, intento y suicidio consumado en varios países por grupos de edad. Es de destacar que en México se registraron 68.4 suicidios consumados en hombres menores de 20 años por cada 100,000 habitantes y que la mayoría de los comportamientos suicidas se presentaron en jóvenes de 11 a 16 años $9,17,10$. En México, en el grupo de 15-19 años, el suicidio es la tercera causa de defunción ${ }^{1}$ 13. En Lituania se presentó la tasa más alta de suicidios consumados en hombres con edad compren- 
Tabla 1. Prevalencia reportada de ideación, intento y suicidio consumado en adolescentes en varios países, según grupos de edad.

\begin{tabular}{|c|c|c|c|c|c|c|}
\hline Año & Autor & Lugar & $\begin{array}{l}\text { Ideación } \\
\%(\mathrm{H} / \mathrm{M})\end{array}$ & $\begin{array}{l}\text { Intento } \\
\%\end{array}$ & $\begin{array}{l}\text { Suicidio consuma- } \\
\text { do*(H/M) }\end{array}$ & $\begin{array}{l}\text { Grupo de } \\
\text { edad }\end{array}$ \\
\hline & & Argentina & - & - & $21.9 / 5.3$ & 15-29 años \\
\hline & & Canadá & - & - & $14.6 / 5.2$ & 15-29 años \\
\hline & & China & - & - & $2.7 / 5.9$ & 15-29 años \\
\hline & & Egipto & - & - & $2.4 / 1.3$ & 15-29 años \\
\hline & & Francia & - & - & $11.8 / 3.2$ & 15-29 años \\
\hline \multirow[t]{6}{*}{2017} & $\begin{array}{l}\text { Abraham Zebib K. y Sher } \\
\text { Leo. }\end{array}$ & India & - & - & $34.9 / 36.1$ & 15-29 años \\
\hline & & Israel & - & - & $7.4 / 1.8$ & 15-29 años \\
\hline & & Japón & - & - & $25.7 / 10.8$ & 15-29 años \\
\hline & & Lituania & - & - & $46.5 / 6.5$ & 15-29 años \\
\hline & & EUA & - & - & $20.4 / 4.7$ & 15-29 años \\
\hline & & México & - & - & $8.9 / 3.1$ & 15-29 años \\
\hline 2017 & Yang Boksun y cols. & República de Corea & 8.2 & - & - & 15-18 años \\
\hline 2017 & Navarro-Gómez N. & España & - & - & $\begin{array}{l}2.6 \\
3.6 / 1.6\end{array}$ & 15-19 años \\
\hline 2017 & Siabato-Macías E. y cols. & Tunja /Colombia & $\begin{array}{l}30 \text { riesgo } \\
20 / 37\end{array}$ & - & - & 13-17 años \\
\hline 2017 & Solaimani-Mohammad A. & Qazvin /Irán & $\begin{array}{l}32.8 \text { ideación } \\
\text { suicida }\end{array}$ & - & - & 14-19 años \\
\hline 2016 & Loboa N.J. y cols. & Tolima /Colombia & 25.7 riesgo & - & - & 11-19 años \\
\hline \multirow[t]{5}{*}{2016} & Romo y cols. & Bolivia & 19.4 riesgo & 27.2 & - & 13-15 años \\
\hline & & Costa Rica & 11.1 & 10.9 & - & 13-15 años \\
\hline & & Honduras & 20.8 & 24.7 & - & 13-15 años \\
\hline & & Perú & 21.2 & 23.7 & - & 13-15 años \\
\hline & & Uruguay & 12.6 & 13.5 & - & 13-15 años \\
\hline 2015 & Hidalgo-Rasmussen y cols. & Jalisco / México & 6.1 & 7.1 & - & 14-18 años \\
\hline 2015 & $\begin{array}{l}\text { Hermosillo-De La Torre } \\
\text { A. y cols. }\end{array}$ & Guanajuato/México & - & $\begin{array}{l}27.8 \text { (fue un } \\
\text { estudio inten- } \\
\text { cionado) }\end{array}$ & - & 13-19 años \\
\hline 2015 & Ceballos-Ospino y cols. & $\begin{array}{l}\text { Santa Martha / } \\
\text { Colombia }\end{array}$ & 25 & - & - & 13-19 años \\
\hline 2015 & $\begin{array}{l}\text { Sánchez-Cervantes F. y } \\
\text { cols. }\end{array}$ & México & - & - & $\begin{array}{l}7 \text { suicidios consumados } \\
68.4 \% / 31.6 \% \\
78.2 \% 15-19 \text { años }\end{array}$ & $<20$ años \\
\hline 2014 & Eguiluz-Romo L. y cols. & $\begin{array}{l}\text { Ciudad de México / } \\
\text { México }\end{array}$ & 21.2 & - & - & $\begin{array}{l}11-15 \text { años } \\
\text { estudiantes }\end{array}$ \\
\hline 2014 & Paniagua S. R. y cols. & Medellín /Colombia & $\begin{array}{l}11.3 \text { orientación } \\
\text { suicida alta } \\
\text { (50.3 media-alta) }\end{array}$ & - & - & $\begin{array}{l}\text { Secundaria y } \\
\text { bachiller }\end{array}$ \\
\hline
\end{tabular}

Fuente: Elaboración propia a partir de varios autores 2018

* Tasa por 100,000 habitantes 
dida entre los 15-29 años (46.5 suicidios por cada 100,000 habitantes de la población en general). La de mujeres se reporta en India (36.1 defunciones, misma constante) entre 15-29 años de edad ${ }^{30}$.

A nivel mundial según varios autores ${ }^{1,11,13,14,25,28 \text {, }}$ 31 , se encuentra entre la segunda y tercera causa de muerte entre los jóvenes de 15-19 años. Mientras que en el grupo de 15-29 años es la tercera causa de muerte ${ }^{13}$.

\section{Factores asociados a suicidio y depresión en ado- lescentes}

Se reporta que el factor con mayor asociación a cualquier conducta suicida es el trastorno depresivo, pero el principal factor determinante del suicidio consumado es el intento de suicidio, aunque previamente está precedida por la ideación suicida $16,14,32,31$. Se ha establecido que la depresión, el intento de acabar con la propia vida y ser varón son variables más frecuentes del suicidio consumado ${ }^{31}$.

En la tabla 2 se destaca que el principal factor de riesgo de comportamientos suicidas y depresión en adolescentes son las malas relaciones con los padres y los problemas entre ellos ${ }^{14,16,32-34}$. Los hombres suelen enmascarar síntomas depresivos con irritabilidad, lo cual origina un subregistro de la depresión al no ser considerada evidencia clínica; también suelen buscar la autoconfirmación de su masculinidad mediante conductas de alto riesgo, entre ellas intentos suicidas ${ }^{35,27}$. Se reporta que las mujeres tienen mayores tasas de intento suicida faIlido $^{27,28}$.

La interacción humana contemporánea está matizada por el uso de tecnología del tipo de redes sociales que afectan de manera distinta a hombres y mujeres, jóvenes y adultos. Su dependencia se asocia a depresión ${ }^{2,36}$. Otras asociaciones son: bajo coeficiente intelectual está relacionado con mayor riesgo de suicidio ${ }^{37}$; quienes viven en áreas urbanas tienen más prevalencia de ideación suicida ${ }^{28}$.

En la tabla 2, se resumen estas condiciones de riego según sexo, cuando los autores lo reportan.

A pesar de la mayor exposición a factores de estrés en adolescentes afroamericanos hay menos frecuencia de depresión que entre anglosajones; además, la autoeficacia se asocia con menor riesgo de presentar trastorno depresivo mayor entre los primeros ${ }^{38}$.

Entre los factores de riesgo menos estudiados están aquellos que asocian depresión y suicidio en la herencia genética. Algunos trabajos reportan bajos niveles de ácido 5-hidroxindolacético (5HIAA), metabolito de la serotonina ( $5 \mathrm{HT})$, detectados en líquido cefalorraquídeo, en personas que han intentado el suicidio violento; además, el gen de la enzima triptófano hidroxilasa (TPH) es el factor limitante para la síntesis de $5 \mathrm{HT}$. Este último tiene 2 isoformas TPH-1 expresada en tejidos periféricos en el cromosoma $11 \mathrm{p} 15$, y la TPH-2 predominante en el cerebro en el cromosoma 12p21 $1^{39}$. Para el gen TPH2 se encontró que el haplotipo GA predispone al intento de suicidio, al igual que el polimorfismo de rs7305115; sin embargo, el haplotipo AT resultó ser un factor protector ${ }^{39}$. No hay evidencia significativa de carga genética para presentar depresión, en las variables de los genes UMAO y 5 HTTLPR $^{8}$.

En la tabla 3 se pueden observar las condiciones de riesgo para la depresión con respecto a la relación con los padres. Dentro de estas condiciones se encuentran: el no vivir con ellos o que éstos tengan una inestabilidad residencial, o sean padres indiferentes a los problemas de los más jóvenes, o bien sean sobrecontroladores o abusivos con hijas e hijos, así como los problemas de pareja, y la violencia intrafamiliar. Estas condicionantes son particularmente trascendentes durante la etapa adolescente donde se requiere de la seguridad y estabilidad familiar.

\section{DISCUSIÓN}

Los estudios revisados muestran claramente la diversidad de elementos individuales, del contexto social, del entorno familiar e incluso de la influencia genética en la asociación depresión y suicidio, en particular en menores de 20 años $8,39,40$. La inseguridad-física, alimentaria, laboral, económica- es la gran ausente en la literatura incluida, probablemente por la dificultad para medirla o por la naturalización elaborada.

Las diferencias en las frecuencias tanto de depresión como suicidio probablemente se deben a los objetivos y propósitos particulares de los estudios revisados, por ende de las características de los participantes. Asimismo, se advierte que no siempre es posible establecer los diagnósticos relacionados con estados depresivos por la confusión asociada culturalmente a efectos hormonales en la adolescencia y/o el estigma de la enfermedad mental. 
Tabla 2. Prevalencia de comportamientos suicidas y condiciones (factores de riesgo) en las que más se presentó según estudios incluidos.

\begin{tabular}{|c|c|c|c|c|c|}
\hline Año & Autor & Lugar/País & Prevalencia & Factores de riesgo & Edad \\
\hline 2017 & $\begin{array}{l}\text { Yang Boksun y } \\
\text { cols. }\end{array}$ & $\begin{array}{l}\text { República de } \\
\text { Corea }\end{array}$ & $8.2 \%$ ideación suicida & $\begin{array}{l}\text { Alteración del ciclo circadiano, depriva- } \\
\text { ción del sueño, insomnio, menos ondas } \\
\delta \text { en el electroencefalograma }\end{array}$ & 15-18 años \\
\hline 2017 & $\begin{array}{l}\text { Siabato-Macías } \\
\text { Elsa y cols. }\end{array}$ & $\begin{array}{l}\text { Tunja/ Co- } \\
\text { lombia }\end{array}$ & $\begin{array}{l}30 \% \text { ideación suicida } \\
\mathrm{H}^{*} 20 \% \mathrm{M}^{*} 37 \% \\
180 \text { suicidios }\end{array}$ & $\begin{array}{l}\text { Sintomatología depresiva } \\
\mathrm{M}^{*} \text { (anhedonia, ánimo negativo) } \\
\mathrm{H}^{*} \text { (autoestima negativa, inefectividad) } \\
\text { Ser mujer }\end{array}$ & $\begin{array}{l}13-17 \text { años } \\
\text { estudiantes }\end{array}$ \\
\hline 2017 & $\begin{array}{l}\text { Solaimani-Mo- } \\
\text { hammad Ali. }\end{array}$ & Qazvin/ Irán & $32.8 \%$ ideación suicida & $\begin{array}{l}\text { Depresión } \\
\text { Falta de autoconfianza y de religiosidad }\end{array}$ & 14-19 años \\
\hline 2016 & $\begin{array}{l}\text { Guerrero-Martí- } \\
\text { nez Luis F. }\end{array}$ & $\begin{array}{l}\text { Medellín/ } \\
\text { Colombia }\end{array}$ & (>5\% no especifica) & $\begin{array}{l}\text { Problemas familiares } \\
\text { Problemas amorosos } \\
\text { Violencia intrafamiliar } \\
\text { Drogas; Abuso sexual }\end{array}$ & $\begin{array}{l}\text { 10-17 años } \\
\text { mujeres }\end{array}$ \\
\hline 2016 & $\begin{array}{l}\text { Romo Matthew } \\
\text { y cols. }\end{array}$ & $\begin{array}{l}\text { Bolivia, } \\
\text { Costa } \\
\text { Rica, Perú, } \\
\text { Uruguay, } \\
\text { Honduras }\end{array}$ & 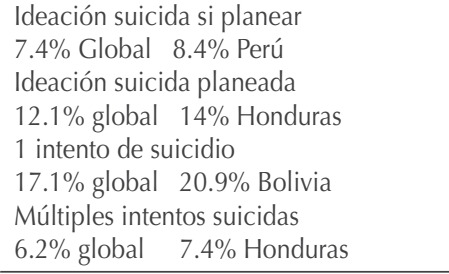 & $\begin{array}{l}\text { Víctima de acoso } \\
\text { Tabaquismo } \\
\text { Ausentismo escolar }\end{array}$ & $\begin{array}{l}10-19 \text { años } \\
\text { estudiantes }\end{array}$ \\
\hline 2016 & $\begin{array}{l}\text { Loboa R. Nelly J. } \\
\text { y cols. }\end{array}$ & $\begin{array}{l}\text { Tolima/ } \\
\text { Colombia }\end{array}$ & $25.7 \%$ ideación suicida & $\begin{array}{l}\text { 11-14 años (suicidio consumado) } \\
\text { Depresión, mala cohesión familiar y } \\
\text { drogas (orientación suicida) }\end{array}$ & 11-19 años \\
\hline 2015 & $\begin{array}{l}\text { Hidalgo-Rasmus- } \\
\text { sen Carlos. }\end{array}$ & $\begin{array}{l}\text { Jalisco / } \\
\text { México }\end{array}$ & $\begin{array}{l}6.1 \% \text { ideación suicida } \\
7.1 \% \text { intento suicida } \\
17.2 \% \text { tristeza o desesperanza }\end{array}$ & $\begin{array}{l}\text { Depresión } \\
\text { Intento suicidio }\end{array}$ & $\begin{array}{l}14-18 \text { años } \\
\text { estudiantes }\end{array}$ \\
\hline 2015 & $\begin{array}{l}\text { Hermosillo De La } \\
\text { Torre Alicia y cols. }\end{array}$ & $\begin{array}{l}\text { Guanajuato } \\
\text { México }\end{array}$ & $\begin{array}{l}27.8 \% \text { intento de suicidio (debido a } \\
\text { que fue un estudio intencionado) }\end{array}$ & $\begin{array}{l}\text { Sintomatología depresiva, drogas } \\
\text { Falta de autocontrol }\end{array}$ & 13-19 años \\
\hline 2015 & $\begin{array}{l}\text { Ceballos Ospino } \\
\text { y cols. }\end{array}$ & $\begin{array}{l}\text { Santa Martha/ } \\
\text { Colombia }\end{array}$ & $\begin{array}{l}\text { Ideación suicida } \\
24 \% \text { riesgo medio } \\
1 \% \text { riesgo alto }\end{array}$ & $\begin{array}{l}\text { Autoestima baja, intentos suicidas, fami- } \\
\text { lia disfuncional, depresión }\end{array}$ & $\begin{array}{l}\text { 13-19 años } \\
\text { Estudiantes }\end{array}$ \\
\hline 2014 & $\begin{array}{l}\text { Sánchez-Loyo y } \\
\text { cols. }\end{array}$ & $\begin{array}{l}\text { Guadalajara/ } \\
\text { México }\end{array}$ & $\begin{array}{l}\text { Muestreo propositivo de } 29 \text { participan- } \\
\text { tes con intento suicida de los cuales } 16 \\
\text { estudiantes se suicidaron }\end{array}$ & $\begin{array}{l}89 \% \text { Violencia intrafamiliar } \\
31 \% \text { problemas de pareja (separación, } \\
\text { violencia) } \\
20 \% \text { sentirse solo }\end{array}$ & 13-18 años \\
\hline 2014 & $\begin{array}{l}\text { Eguiluz-Romo } \\
\text { Luz y cols. }\end{array}$ & $\begin{array}{l}\text { Ciudad de } \\
\text { México/ } \\
\text { México }\end{array}$ & $21.2 \%$ ideación suicida & $\begin{array}{l}\text { Depresión } \\
\text { Poca comunicación y afecto de los } \\
\text { padres }\end{array}$ & $\begin{array}{l}11-15 \text { años } \\
\text { estudiantes }\end{array}$ \\
\hline 2014 & $\begin{array}{l}\text { Paniagua S. } \\
\text { Ramón y cols. }\end{array}$ & $\begin{array}{l}\text { Medellín / } \\
\text { Colombia }\end{array}$ & $\begin{array}{l}11.3 \% \text { orientación suicida alta } \\
(50.3 \% \text { media-alta })\end{array}$ & $\begin{array}{l}\text { Sintomatología depresiva (Ánimo y auto- } \\
\text { estima negativas, anhedonia, inefectivi- } \\
\text { dad), vulnerabilidad en valores y apoyo, } \\
\text { 11-14 años, problemas familiares }\end{array}$ & $\begin{array}{l}\text { Secundaria } \\
\text { y bachiller }\end{array}$ \\
\hline $\begin{array}{l}1998 \\
- \\
2011\end{array}$ & $\begin{array}{l}\text { Sánchez-Cervan- } \\
\text { tes Felipe y cols. }\end{array}$ & México & $\begin{array}{l}\text { 7/100,000 hab suicidio culminado } \\
H^{*} 68.4 \% \quad M^{*} 31.6 \% \\
78.2 \% 15-19 \text { años }\end{array}$ & $\begin{array}{l}\text { La pérdida de un ser querido, divorcio de } \\
\text { padres, trastornos psiquiátricos, abusos } \\
\text { en la niñez } \\
\text { Estado de México } \\
\text { Hombre }\end{array}$ & <20 años, \\
\hline
\end{tabular}

Fuente: Elaboración propia a partir de varios autores $2017 .{ }^{*} \mathrm{H}=$ hombres, $\mathrm{M}=$ mujeres 
Tabla 3. Prevalencia de depresión y condiciones de riesgo según edad, según los estudios analizados.

\begin{tabular}{|c|c|c|c|c|c|}
\hline Año & Autor & Lugar/país & Prevalencia & Condiciones de riesgo & Edades \\
\hline 2017 & $\begin{array}{l}\text { Burdzovic-An- } \\
\text { dreas Jasmina y } \\
\text { cols. }\end{array}$ & Oslo / Noruega & $\begin{array}{l}\text { Trastorno depresivo mayor } \\
\mathrm{H}^{*} 2-6 \% \quad \mathrm{M}^{*} 8.5 \%\end{array}$ & $\begin{array}{l}\text { No vivir con padres biológicos } \\
\text { Enfermedad crónica } \\
\text { Insatisfacción escolar } \\
\text { Falta de amigos } \\
\text { Inestabilidad residencial }\end{array}$ & $\begin{array}{l}\text { Secundaria } \\
\text { y bachiller }\end{array}$ \\
\hline 2017 & $\begin{array}{l}\text { Yang Boksun y } \\
\text { cols. }\end{array}$ & $\begin{array}{l}\text { República de } \\
\text { Corea }\end{array}$ & 3.5\% depresión severa & $\begin{array}{l}\text { Alteración del ciclo circadiano, deprivación } \\
\text { del sueño, insomnio, }\end{array}$ & 15-18 años \\
\hline 2017 & $\begin{array}{l}\text { Kiat-AngJin y } \\
\text { cols. }\end{array}$ & $\begin{array}{l}\text { Serdang / } \\
\text { Malasia }\end{array}$ & $\begin{array}{l}\text { Depresión } \\
14 \% \text { severa } \\
18 \% \text { moderada } \\
36 \% \text { leve }\end{array}$ & $\begin{array}{l}\text { Padres indiferentes, abusivos, sobre contro- } \\
\text { ladores } \\
\text { Mujer }\end{array}$ & $\begin{array}{l}13-17 \text { años } \\
\text { estudiantes }\end{array}$ \\
\hline 2017 & $\begin{array}{l}\text { Siabato-Macías } \\
\text { Elsa y cols. }\end{array}$ & $\begin{array}{l}\text { Tunja /Colom- } \\
\text { bia }\end{array}$ & $\begin{array}{l}\text { Depresión } \\
\text { Global } 9 \% \quad H^{*} 5 \% \quad M^{*} 12 \%\end{array}$ & Mujer & 13-17 años \\
\hline 2016 & $\begin{array}{l}\text { Vicente Benja- } \\
\text { mín y cols. }\end{array}$ & $\begin{array}{l}\text { Concepción / } \\
\text { Chile }\end{array}$ & 10\% depresión & $\begin{array}{l}\text { Problemas de pareja } \\
\text { Mujer }\end{array}$ & 18-75 años \\
\hline 2016 & Ressett Santiago. & $\begin{array}{l}\text { Entre Ríos / } \\
\text { Argentina }\end{array}$ & 11\% depresión & $\begin{array}{l}\text { Mala relación con los progenitores (principal- } \\
\text { mente la madre) } \\
\text { Mujer }\end{array}$ & $\begin{array}{l}12-18 \text { años } \\
\text { estudiantes }\end{array}$ \\
\hline 2015 & $\begin{array}{l}\text { Gonzálvez } \\
\text { María y cols. }\end{array}$ & $\begin{array}{l}\text { Alicante / } \\
\text { España }\end{array}$ & $\begin{array}{l}\text { Sintomatología depresiva } \\
\text { fumadores } \\
\text { Estado de ánimo } \\
\text { Medio } 19.9 \% \\
\text { Bajo } 10.4 \% \\
\end{array}$ & $\begin{array}{l}\text { Tabaco mayor consumo a mayor edad y ser } \\
\text { mujer } \\
\text { Drogas }\end{array}$ & $\begin{array}{l}14-19 \text { años } \\
\text { estudiantes }\end{array}$ \\
\hline 2015 & $\begin{array}{l}\text { Rivera Rivera } \\
\text { Leonor y cols. }\end{array}$ & $\begin{array}{l}\text { Cuernavaca / } \\
\text { México }\end{array}$ & $\begin{array}{l}\text { Depresión } \\
\text { Global } 27 \% \\
\mathrm{H}^{*} 18 \% \mathrm{M}^{*} 34 \%\end{array}$ & $\begin{array}{l}64 \% \text { autoestima baja } \\
58 \% \text { alcohol } \\
20 \% \text { tabaco } \\
11 \% \text { drogas } \\
60 \% \text { violencia intrafamiliar } \\
\text { Poca comunicación con padres } \\
\text { Mujer }\end{array}$ & $\begin{array}{l}14-19 \text { años } \\
\text { estudiantes }\end{array}$ \\
\hline 2015 & $\begin{array}{l}\text { Ceballos-Ospino } \\
\text { y cols. }\end{array}$ & $\begin{array}{l}\text { Santa Martha / } \\
\text { Colombia }\end{array}$ & $\begin{array}{l}\text { Sintomatología depresiva } \\
\text { Leve } 5 \% \\
\text { Moderada } 7 \% \\
\text { Grave } 4 \%\end{array}$ & $\begin{array}{l}\text { Autoestima baja } \\
\text { Mujer }\end{array}$ & $\begin{array}{l}\text { 13-19 años } \\
\text { estudiantes }\end{array}$ \\
\hline 2015 & $\begin{array}{l}\text { González-Forte- } \\
\text { za y cols. }\end{array}$ & $\begin{array}{l}\text { Estado de } \\
\text { Michoacán y } \\
\text { Ciudad de } \\
\text { México / } \\
\text { México }\end{array}$ & $\begin{array}{l}\text { Sintomatología depresiva } \\
\text { Global } 12.4 \% \quad \mathrm{H}^{*} 7.7 \% \quad \mathrm{M}^{*} \\
17.8 \% \\
\text { Depresión subclínica } \\
\text { Global } 27.3 \% \mathrm{H}^{*} 28.5 \% \mathrm{M}^{*} \\
25.9 \%\end{array}$ & Mujer & $\begin{array}{l}\text { Estudiantes } \\
\text { de secun- } \\
\text { daria }\end{array}$ \\
\hline 2014 & $\begin{array}{l}\text { Rodríguez-Puen- } \\
\text { tes Adriana } \\
\text { Paola y cols. }\end{array}$ & $\begin{array}{l}\text { Bogotá / } \\
\text { Colombia }\end{array}$ & $27.5 \%$ depresión & $\begin{array}{l}\text { Dependencia de internet (uso de las redes } \\
\text { sociales) } \\
\text { Mujer }\end{array}$ & $\begin{array}{l}\text { 11-15 años } \\
\text { de edad }\end{array}$ \\
\hline 2014 & $\begin{array}{l}\text { Eguiluz-Romo } \\
\text { Luz de los Ánge- } \\
\text { les y cols. }\end{array}$ & $\begin{array}{l}\text { Ciudad de Mé- } \\
\text { xico /México }\end{array}$ & $\begin{array}{l}17.8 \% \text { depresión leve } \\
7.5 \% \text { depresión moderada } \\
3 \% \text { depresión severa }\end{array}$ & $\begin{array}{l}\text { Poca comunicación y afectividad funcional } \\
\text { familiar } \\
\text { Mujer }\end{array}$ & 11-15 años \\
\hline 2014 & $\begin{array}{l}\text { Rosa-Alcázar y } \\
\text { cols. }\end{array}$ & $\begin{array}{l}\text { Murcia y Casti- } \\
\text { Ila-La Mancha / } \\
\text { España }\end{array}$ & Depresión 26.7\% & $\begin{array}{l}\text { Autoestima baja } \\
\text { Mujer }\end{array}$ & $\begin{array}{l}12-18 \text { años } \\
\text { estudiantes }\end{array}$ \\
\hline
\end{tabular}




\begin{tabular}{|c|c|c|c|c|c|}
\hline 2014 & $\begin{array}{l}\text { Murillo-Pérez } \\
\text { Luis y cols. }\end{array}$ & Lima /Perú & $\begin{array}{l}\text { Trastorno depresivo Mayor } \\
4.2 \%\end{array}$ & $\begin{array}{l}6.3 \% \text { Actividad física baja } \\
16 \% \text { Desempleo } \\
6.1 \% \text { mujer } \\
\text { Dormir }<6 \text { hrs }\end{array}$ & $\begin{array}{l}\text { Universita- } \\
\text { rios } 25-33 \\
\text { años }\end{array}$ \\
\hline 2013 & Berenzon y cols. & México & $\begin{array}{l}\text { Trastorno depresivo mayor } \\
\text { Global } 9.2 \% \mathrm{H}^{*} 5.4 \% \\
\mathrm{M}^{*} 10.4 \%\end{array}$ & $\begin{array}{l}\text { Ama de casa, Cuidar algún enfermo, pobreza, } \\
\text { Desempleo, } \\
\text { Aislamiento, problemas legales; violencia, } \\
\text { drogas, } \\
\text { Mujer }\end{array}$ & $\begin{array}{l}\text { Población } \\
\text { en general }\end{array}$ \\
\hline
\end{tabular}

Fuente: Elaboración propia a partir de varios autores 2017.

$* \mathrm{H}=$ hombres, $\mathrm{M}=$ mujeres

Por la naturaleza del fenómeno, los estudios sobre suicidio no tienen diseños que permitan identificar de forma incuestionable el peso específico de los elementos involucrados en la causalidad. Lo que parece claro es que entre la ideación y el suicidio consumado hay un rango amplio de variabilidad y no todas las veces el plan o intento cumplen con su objetivo; sin embargo, de acuerdo con Eguiluz ${ }^{16}$, Sánchez $^{34}{ }^{4}$ Guerrero $^{32}$ y Dávila ${ }^{31}$ el intento y la ideación suicida son componentes indispensables para concluir fatalmente. Las tasas de suicidio en el periodo 2007-2011 en Andalucía España fueron mayores a mayor altitud, mayor privación material y poblaciones más pequeñas ${ }^{23}$. Este último podría ser un factor relacionado para discutir en estudios posteriores. Esta condición variable se relaciona también con la residencia en zonas urbanas de quienes atentan contra su vida, debido a que, como OMS señala, el estigma del fenómeno resulta un obstáculo para el registro.

Según Agudelo, el subregistro de la depresión en hombres puede deberse a razones del deber ser hombre -implicaciones de género- que explicaría la más alta prevalencia de suicidios en hombres, a pesar de disponer de recursos más eficaces para lidiar con emociones negativas, reportando menor prevalencia de depresión ${ }^{35}$.

Respecto a los polimorfismos encontrados en el estudio, conviene señalar que se evaluaron en una población en la que se desconocía si tomaban algún tipo de antidepresivo; aun así, la presencia del polimorfismo rs7305115 y el haplotipo GA del gen TPH-2 resultan positivos en quienes ya habían intentado el suicidio anteriormente ${ }^{39}$. Se trata de un factor de riesgo no modificable, aunque puede ser empleado para la consejería genética.

Entre las limitaciones de este trabajo se identifican: (1) la selección de artículos que se enfocan únicamente en acciones previas al suicidio consumado, como intento suicida o ideación suicida, sin determinar si se consumó; (2) en otros casos algunos artículos no distinguen entre orientación y/o comportamiento y/o conducta suicida, en la metodología y/o instrumentos para evaluar depresión y las características clínicas de los participantes; (3) se consideró un área poblacional muy amplia con muy pocos casos; (4) para evaluar la depresión a nivel mundial se tomaron en cuenta artículos que abordaban diversos aspectos del proceso; (5) los artículos tratan de adolescentes con diferentes rangos de edad por lo que puede haber matices en los resultados, dificultando una conclusión contundente. Es indispensable que las cifras disponibles se interpreten y comparen con cautela.

Debido a que no se registra lo que no se conoce, es posible que haya una mayor tasa de suicidio en adolescentes, si consideramos que existe una cada vez más pobre comunicación paterno-filial: necesidad de los padres de emplearse en dos o más trabajos y uso indiscriminado de las nuevas tecnologías, lo que ayuda a omitir lo que callan los adolescentes, aunado a la dificultad reportada para pedir ayuda.

Desde la calidad de la crianza, la herencia genética, el ambiente social más complejo, la geografía, los rasgos de personalidad hasta profesar creencias religiosas, son elementos a considerar en la atención de esta problemática. Los jóvenes llevan a cabo actos de violencia máxima contra sí mismos, es decir, devuelven la agresión a una sociedad hostil. Las políticas públicas y las estrategias de intervención deben considerar seriamente estos orígenes estructurales.

Es cardinal destacar el papel que puede/debe desempeñar la familia como el primer espacio de contención para los adolescentes, sin dejar de lado la participación de los profesores en las escuelas 
en donde la convivencia con otros chicos y chicas puede ser un desafío determinante para la sociedad. Resulta prioritario desde temprana edad fortalecer la autoestima, el manejo de las emociones y la frustración, en las que subyacen múltiples eventos. No menos importante también es el reconocimiento positivo de aquellas situaciones en donde los adolescentes logran comportamientos asertivos ante situaciones que lo ameritan en su vida cotidiana.

Lo que resulta indispensable es visibilizar la asociación entre suicidio y depresión en adolescentes, para replantear estrategias de prevención y atención en los distintos ámbitos de acción de los jóvenes, procurar un ambiente promotor de salud física, social y mental.

Agradecimiento: Los autores agradecen al Cuerpo Académico Promoción y Educación en Salud (CA 042-UNACH) los recursos materiales, humanos y financieros recibidos para la realización de este trabajo.

Conflictos de interés: Los autores declaran no tener conflicto alguno de intereses.

\section{REFERENCIAS BIBLIOGRÁFICAS}

1. Hidalgo Vicario MI, Ceñal González Fierro MJ, Güemes Hidalgo M. La adolescencia. Aspectos físicos, psicosociales y médicos. Principales cuadros clínicos. Med - Programa Form Médica Contin Acreditado. 2014; 11(61):3579-87.

2. Rodríguez Puentes AP, Fernández Parra A. Relación entre el tiempo de uso de las redes sociales en internet y la salud mental en adolescentes colombianos. Acta Colomb Psicol. 2014:131-40.

3. Grist R, Porter J, Stallard P. Mental Health Mobile Apps for Preadolescents and Adolescents: A Systematic Review. J Med Internet Res. 2017;19(5):e176.

4. Soleimani MA, Pahlevan Sharif S, Bahrami N, Yaghoobzadeh A, Allen KA, Mohammadi S. The relationship between anxiety, depression and risk behaviors in adolescents. Int J Adolesc Med Health [en línea]. 2017 Enero [consultado el 13 abril 2018]. Disponible en: http://www.degruyter.com/view/j/ijamh.ahead-ofprint/ijamh-2016-0148/ijamh-2016-0148.xml

5. Bi B, Liu W, Zhou D, Fu X, Qin X, Wu J. Personality traits and suicide attempts with and without psychiatric disorders: analysis of impulsivity and neuroticism. BMC Psychiatry [en línea]. 2017 [consultado el 13 de abril de 2018];17(1). Disponible en: http://bmcpsy- chiatry.biomedcentral.com/articles/10.1186/s12888017-1453-5

6. Yang B, Choe K, Park Y, Kang Y. Associations among daytime sleepiness, depression and suicidal ideation in Korean adolescents. Int J Adolesc Med Health [en línea]. 2017 [consultado el 13 abril 2018]. Disponible en: http://www.degruyter.com/view/j/ijamh.ahead-ofprint/ijamh-2016-0161/ijamh-2016-0161.xml

7. de Dios C, Goikolea JM, Colom F, Moreno C, Vieta E. Los trastornos bipolares en las nuevas clasificaciones: DSM-5 y CIE-11. Rev Psiquiatr Salud Ment. 2014;7(4):179-85

8. Vicente P B, Rojas P R, Saldivia B S, Pérez V C, MelipiIlán A R, Hormazábal P N, et al. Determinantes biopsicosociales de depresión en pacientes atendidos en Centros de Atención Primaria de Concepción, Chile. Rev Chil Neuro-Psiquiatr. 2016; 54(2):102-12.

9. Loboa NJ, Morales DF, Clínica Tolima. Perfil de orientación al suicidio en adolescentes escolarizados, ViIlahermosa - Tolima, 2013. Rev Fac Nac Salud Pública [en línea]. 2016 [consultado el 13 abril 2018];34(1). Disponible en: http://aprendeenlinea.udea.edu.co/revistas/index.php/fnsp/article/view/19885

10. Guerrero-Martínez, LF. Descripción del intento de suicidio como constructo de violencia en la población pediátrica femenina. Rev Fac Nac Salud Pública [en línea]. 2016 [consultado el 13 abril 2018];34(3):306315. Disponible en: http://www.redalyc.org/articulo. oa? id=12046949005.

11. Madariaga A C, Ulloa D O, Gómez V A-G, Iriondo C P, Alvarado M R. Muertes por suicidio en la Región de Tarapacá, años 1990-2013. Rev Chil Neuro-Psiquiatr. Septiembre de 2016;54(3):250-8.

12. Corona Miranda, B, Hernández Sánchez, M, García Pérez, RM. Mortalidad por suicidio, factores de riesgos y protectores. Rev Haban Cien Méd [en línea]. 2016 [consultado el 13 abril 2018];15(1):90100. Disponible en: http://www.redalyc.org/articulo. oa?id=180444213011.

13. Navarro-Gómez N. El suicidio en jóvenes en España: cifras y posibles causas. Análisis de los últimos datos disponibles. Clínica Salud. 2017;28(1):25-31.

14. Sánchez-Cervantes FS, Serrano-González RE, Márquez-Caraveo ME. Suicidios en menores de 20 años. México 1998-2011. Salud Mental. 2015;38(5):37989.

15. Siabato Macías, EF, Forero Mendoza, IX, Salamanca Camargo, Y. Asociación entre depresión e ideación suicida en un grupo de adolescentes colombianos. 
Pensam Psicol [en línea]. 2017 [consultado el 13 abril 2018]; 15(1):51-61. Disponible en: http://www. redalyc.org/articulo.oa? $\mathrm{id}=80149351004$

16. Eguiluz Romo, LdL, Ayala Mira, M. Relación entre ideación suicida, depresión y funcionamiento familiar en adolescentes. Psicol Iberoam [en línea]. 2014 [consultado el 13 abril 2018];22(2):72-80. Disponible en: http://www.redalyc.org/articulo. oa? id=133938134009.

17. Paniagua S., RE, González P., CM, Rueda R., SM. Orientación al suicidio en adolescentes en una zona de Medellín, Colombia. Rev Fac Nac Salud Pública [en línea]. 2014 [consultado el 12 abril 2018]; 32(3):314-321. Disponible en: http://www.redalyc. org/articulo.oa?id=12031816008.

18. Dolphin L, Hennessy E. Labelling effects and adolescent responses to peers with depression: an experimental investigation. BMC Psychiatry [en línea]. 2017 Dic [consultado el 13 abril 2018];17(1). Disponible en: http://bmcpsychiatry.biomedcentral.com/articles/10.1186/s12888-017-1389-9

19. Romo ML, Kelvin EA. Impact of bullying victimization on suicide and negative health behaviors among adolescents in Latin America. Rev Panam Salud Pública. 2016;40(5):347-355

20. Vivian da Cunha, R, Barreyro, JP. Revisión del estado del arte de la depresión, la ansiedad y el apoyo social en torno del tema de niños y adolescentes institucionalizados. Subjetividad y Procesos Cognitivos [en línea]. 2015 [consultado el 13 abril 2018];19(2):5873. Recuperado de: http://www.redalyc.org/articulo. oa? id=339643529003.

21. Rivera-Rivera L, Rivera-Hernández P, Pérez-Amezcua B, Leyva-López A, Castro F de. Factores individuales y familiares asociados con sintomatología depresiva en adolescentes de escuelas públicas de México. Salud Pública Méx. 2015;57(3):219.

22. Berenzon S, Lara MA, Robles R, Medina-Mora ME. Depresión: estado del conocimiento y la necesidad de políticas públicas y planes de acción en México. Salud Pública Méx. 2013; 55(1):74-80.

23. Alameda-Palacios J, Ruiz-Ramos M, García-Robredo B. Mortalidad por suicidio en Andalucía: distribución geográfica y relación con el uso de antidepresivos, la altitud y desigualdades socioeconómicas. Rev Esp Salud Pública. 2015; 89(3):283-93.

24. Martínez-Aguayo JC, Arancibia M M, Silva I H. Psicofarmacología del suicidio: un análisis crítico. Rev Chil Neuro-Psiquiatr. 2015; 53(2):127-33.
25. Hermosillo-De la Torre, AE, Vacio Muro, MdlÁ, Méndez-Sánchez, C, Palacios Salas, P, Sahagún Padilla, MÁ. Sintomatología depresiva, desesperanza y recursos psicológicos: una relación con la tentativa de suicidio en una muestra de adolescentes mexicanos. Acta Universitaria [en línea]. 2015; 25(2):5256. Recuperado de: http://www.redalyc.org/articulo. oa? id=41648310009

26. Arrona-Palacios, A, García, A, Villarreal-Sotelo, K, Durán, H, Retana, A. Prevalencia de suicidios consumados y su análisis evolutivo en Reynosa, Tamaulipas. CienciaUAT [en línea]. 2009; 4(1):8-12. Recuperado de: http://www.redalyc.org/articulo. oa?id=441942917008.

27. González-Forteza C, Torre AEH de la, Vacio-Muro M de los Á, Peralta R, Wagner FA. Depresión en adolescentes. Un problema oculto para la salud pública y la práctica clínica. Bol Méd Hosp Infant México. 2015; 72 (2):149-55.

28. The U.S. Caribbean Alliance for Health Disparities Research Group (USCAHDR), Brown CR, Hambleton IR, Sobers-Grannum N, Hercules SM, Unwin N, et al. Social determinants of depression and suicidal behaviour in the Caribbean: a systematic review. BMC Public Health [en línea]. 2017; [consultado el 13 abril 2018];17(1). Disponible en: http://bmcpublichealth. biomedcentral.com/articles/10.1186/s12889-0174371-z

29. Hidalgo-Rasmussen C, Martín AH-S. Comportamientos de riesgo de suicidio y calidad de vida, por género, en adolescentes mexicanos, estudiantes de preparatoria. Ciênc Saúde Coletiva. 2015; 20(11):3437-45.

30. Abraham ZK, Sher L. Adolescent suicide as a global public health issue. Int J Adolesc Med Health [en línea]. Enero de 2017 [consultado el 13 de abril de 2018]. Disponible en: http://www.degruyter. com/view/j/ijamh.ahead-of-print/ijamh-2017-0036/ ijamh-2017-0036.xml

31. Dávila CA, Pardo AM. Mortalidad por suicidios en Colombia y México: tendencias e impacto entre 2000 y 2013. Biomédica [en línea]. 2016 Abril [consultado el 13 abril 2018];36(3). Disponible en: http://www. revistabiomedica.org/index.php/biomedica/article/ view/3224

32. Guerrero-Martínez, LF. Descripción del intento de suicidio como constructo de violencia en la población pediátrica femenina. Rev Fac Nac Salud Pública [en línea]. 2016 [consultado el 13 abril 2018];34(3):306315. Disponible en: http://www.redalyc.org/articulo. oa? id=12046949005 
33. Ceballos-Ospino GA, Suarez-Colorado Y, Suescún-Arregocés J, Gamarra-Vega LM, González KE, Sotelo-Manjarres AP. Ideación suicida, depresión y autoestima en adolescentes escolares de Santa Marta. Duazary. 2015;12(1):15.

34. Sánchez-Loyo LM, Morfín-López T, García de Alba JE, Quintanilla-Montoya R, Hernández-Millán R. et al. Intento de Suicidio en Adolescentes Mexicanos: Perspectiva desde el Consenso Cultural. Acta Investig Psicológica. 2014;4(1):1446-58.

35. Agudelo Vélez DM, Gómez Maquet Y, López PL. Propiedades Psicométricas del Inventario de Depresión Estado Rasgo (IDER) con una muestra de población general colombiana. Av Psicol Latinoam. 2014;32(1):7184.

36. Arab LE, Díaz GA. Impacto de las redes sociales e internet en la adolescencia: aspectos positivos y negativos. Rev Méd Clín Las Condes. 2015;26(1):7-13.
37. Dutta R, Ball HA, Siribaddana SH, Sumathipala A, Samaraweera S, McGuffin P, et al. Genetic and other risk factors for suicidal ideation and the relationship with depression. Psychol Med. 2017;47(14):2438-49.

38. Assari S, Caldwell C. The Link between Mastery and Depression among Black Adolescents; Ethnic and Gender Differences. Behav Sci. 2017;7(4):32.

39. López-Narváez ML, Tovilla-Zárate CA, González-Castro TB, Juárez-Rojop I, Pool-García S, Genis A, et al. Association analysis of TPH-1 and TPH-2 genes with suicidal behavior in patients with attempted suicide in Mexican population. Compr Psychiatry. 2015; 61:727.

40. Patel V, Lund C, Hatherill S, Plagerson S, Corrigall J, Funk M, Flisher A. Mental disorders: equity and social determinants. En: Erik Blas and Anand Sivasankara Kurup (Edits) WHO: Equity, social determinants and public health programmes. Switzerland: WHO;2010. p. 115-137. 